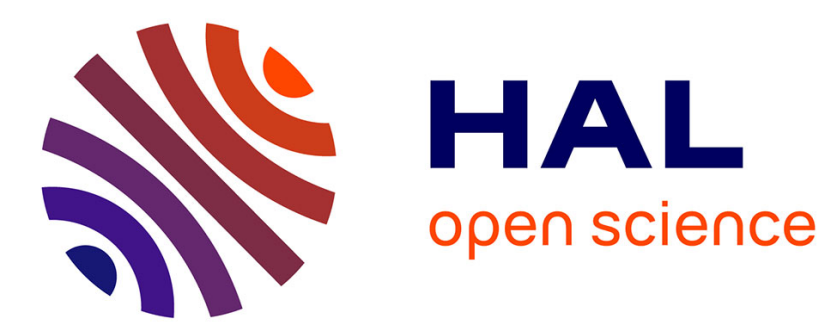

\title{
La lutte contre le non-recours: des enjeux pour la production des politiques sociales
}

Philippe Warin, Pierre Mazet

\section{To cite this version:}

Philippe Warin, Pierre Mazet. La lutte contre le non-recours: des enjeux pour la production des politiques sociales. Regards, 2014, Actualités de l'accès aux droits, 46, pp.75-82. hal-01099957

\section{HAL Id: hal-01099957 \\ https://hal.science/hal-01099957}

Submitted on 29 Nov 2019

HAL is a multi-disciplinary open access archive for the deposit and dissemination of scientific research documents, whether they are published or not. The documents may come from teaching and research institutions in France or abroad, or from public or private research centers.
L'archive ouverte pluridisciplinaire HAL, est destinée au dépôt et à la diffusion de documents scientifiques de niveau recherche, publiés ou non, émanant des établissements d'enseignement et de recherche français ou étrangers, des laboratoires publics ou privés. 


\title{
La lutte contre le non-recours : des enjeux pour la production des politiques sociales
}

\author{
Philippe Warin \\ Directeur de recherche au CNRS et enseignant à Sciences-Po Grenoble, il a cofondé l'observatoire \\ des non-recours aux droits et services (ODENORE). Ses travaux portent sur la réception des \\ politiques par les publics et questionnent les notions de pertinence de l'offre et d'adhésion à l'offre.
}

Pierre Mazet

Chargé de recherche à l'ODENORE et enseignant à Sciences-Po Grenoble, il mène des travaux sur le phénomène du non-recours appréhendé sous l'angle des comportements individuels et collectifs en situations normatives.

La question du non-recours aux droits et services est posée sur différents agendas politiques et institutionnels. Le Plan pluriannuel de lutte contre la pauvreté et pour l'inclusion sociale présenté par le gouvernement le 21 janvier 2013 en tient compte directement dans ses orientations pour venir en aide aux personnes les plus démunies et les accompagner vers l'autonomie. Les nouvelles conventions d'objectifs et de gestion des organismes sociaux inscrivent ou réinscrivent la question au cœur de leurs priorités. Au niveau des collectivités locales, la recherche de solutions est souvent innovante.

Ce contexte général incite à s'interroger sur les enjeux de la lutte contre le non-recours pour la production des politiques sociales. Le point de vue présenté ici est celui de l'Observatoire des nonrecours aux droits et services (ODENORE) qui, modestement, a contribué depuis 2002 à faire reconnaître le phénomène. II s'agit donc d'un propos situé. Celui-ci n'est en rien exhaustif et surtout ne cherche pas à dire à la place des acteurs ce que les enjeux de la lutte contre le non-recours devraient être.

Pour percevoir les enjeux de la lutte contre le non-recours pour la production même des politiques, il convient de se demander de quoi le non-recours est-il la critique ? Répondre à cette question permet de discuter de ce que la lutte contre le non-recours aurait à changer : ces changements étant ses enjeux.

La littérature internationale fait apparaître le non-recours comme la " contradiction » même du ciblage qui organise l'accès aux droits [Conning, Kevane, 2002 ; Gwatkin, 2000 ; Sen 1995]. C'est en partant de l'observation de taux de non-recours plus élevés lorsque les prestations sont ciblées que l'explication institutionnelle des obstacles dans l'accès aux droits sociaux est venue contrebalancer les premières explications comportementales qui renvoyaient le non-recours aux manques et incapacités des individus. Du coup, la critique est aussi directe que la relation entre ciblage et non-recours paraît mécanique. Et la solution l'est tout autant : pour éviter le non-recours, il faudrait sortir des politiques ciblées. Des acteurs avertis vont dans ce sens et s'interrogent publiquement sur la nécessité de revenir à davantage d'universalité dans la construction des droits sociaux, mais sans dire généralement comment.

Cela étant, il n'est pas certain que la critique du non-recours s'adresse en premier au ciblage, qui est un ensemble de critères d'éligibilité (niveau de ressources, âge, handicap, degré de formation, quartiers défavorisés, etc.) et de modalités techniques de mise en œuvre des prestations (dispositif spécifique pour une population donnée, mesure de sélectivité dans l'accès aux ressources, différenciation tarifaire, modulation du niveau d'une prestation financière en fonction de critères particuliers, mécanisme d'individualisation de l'offre selon la situation ou le comportement du bénéficiaire). En effet, si l'on rassemble les griefs auxquels conduisent les faits et les chiffres sur le non-recours [Odenore, 2013], ce sont d'autres critiques, avec d'autres implications, qui apparaissent, portant notamment sur le manque de légitimité des normes centrales et sur la complexité de l'environnement institutionnel et réglementaire. 


\section{Renforcer la légitimité des normes des politiques sociales}

Parmi les formes de non-recours, la non demande mérite l'attention. Outre les calculs individuels de type coût-avantage, cette forme de non-recours indique dans bon nombre de cas l'existence de conflits de représentations sur la justesse même de la prestation. Celle-ci heurte les destinataires quand la norme sociale qu'elle contient ne leur paraît pas acceptable. Ce ne sont pas principalement les critères ou techniques du ciblage qui créent la non demande, mais « le motif » de la prestation. Celui-ci est exposé dans un ou des textes (loi, décret, arrêté, circulaire, etc.) qui donnent à la prestation son assise juridique. Or, les motifs ne paraissent pas nécessairement acceptables. Si bien que tout en étant fondées en droit, il peut manquer aux prestations sociales, notamment aux minima sociaux, la légitimité que seule l'adhésion des ouvrants-droit potentiels peut leur conférer, et non seulement celle des catégories non bénéficiaires qui les financent notamment par l'impôt.

L'évaluation du Revenu de Solidarité Active (RSA) entré en vigueur le $1^{\text {er }}$ juin 2009 permet d'illustrer en quoi le non-recours à un minimum social peut être une critique du manque de légitimité des normes centrales. Avec un taux de non-recours au RSA-activité estimé à $68 \%$, le Comité national d'évaluation a avancé dans son rapport que les travailleurs pauvres désertent le dispositif. Des analyses ont mis en avant des raisons individuelles, renvoyant l'explication à une certaine méconnaissance du dispositif et à ses conditions pour près des deux-tiers des non-recourants n'ayant jamais bénéficié de la prestation, mais connaissant son existence et se considérant éligibles [Domingo, 2012]. II en reste donc au moins un tiers qui déclare ne pas être intéressé par le RSAactivité ; ce qui laisse soupçonner "un défaut congénital du dispositif " pour reprendre l'expression d'Evelyne Serverin dans son analyse du non-recours [Serverin, 2012]. Ce résultat renvoie directement au motif du projet de loi qui fixe au RSA-activité l'objectif de « faire des revenus du travail le socle des ressources des individus ". L'aide apportée venant consolider des bas revenus afin que les salariés restent en activité, il n'est pas exclu qu'une partie des non-recourants récuse un dispositif d'intéressement permanent qui institue le travail précaire comme norme sociale. Par hypothèse, des ouvrants-droit potentiels du RSA-activité peuvent attendre avant tout du gouvernement une politique des salaires qui leur permettrait de vivre dignement de leur travail, au lieu d'être suspendus à l'aide publique.

Ce non-recours par non demande et cette non demande par manque d'adhésion sont, d'une même pièce, la conséquence directe d'un mode de production des politiques sans leurs publics, de définition de la demande sociale sans les citoyens. Pareille situation pose fondamentalement la question de la production de l'assentiment dans les politiques publiques, que des travaux collectifs ont cherché à mettre en évidence, comme ce fût le cas en France à l'époque où le gouvernement venait de mettre en place une politique d'évaluation des politiques publiques. Cela fait des dizaines d'années que des réformes tentent d'introduire la participation des bénéficiaires dans la construction des politiques. L'analyse du non-recours montre, s'il le fallait, l'utilité de cette participation pour prévenir des désaccords difficiles à redresser ensuite, qui, laissés sans réponse, peuvent entamer la confiance des citoyens dans les institutions ${ }^{1}$. Aussi, est-ce la question politique et institutionnelle des instances et dispositifs de participation des destinataires des politiques qui s'impose à nouveau, notamment en pensant le non-recours comme critère (sinon comme référentiel) d'évaluation [Milet, Warin, 2014].

\section{Sécuriser l'application des droits}

En même temps, la complexité de l'environnement institutionnel et réglementaire des dispositifs d'action montre, cette fois sur le plan de la réalisation des politiques et non seulement de leur construction, que la critique du non-recours ne s'arrête pas aux seules conditions du ciblage.

La multiplication des règles formelles portant sur l'éligibilité, les exigences, contraintes ou réquisits imposés au demandeur en matière de rendez-vous à honorer, de documents à fournir (en particulier lorsque les demandeurs ne sont pas en possession des documents et qu'ils doivent les demander à

\footnotetext{
${ }^{1}$ Avant impôts et prestations sociales, le rapport entre le niveau de vie des $10 \%$ des plus aisés et des $10 \%$ les plus modestes est de 17,6 . Après redistribution, ce rapport n'est plus que de $5,7 \%$ selon I'INSEE. Baisser le niveau des prestations (en laissant filer le non-recours notamment) ne peut donc que creuser les inégalités entre catégories sociales ; ce qui n'est pas forcément bon pour le sentiment de justice sociale et la cohésion sociale, et in fine pour la confiance dans les institutions.
} 
une autre administration), etc., mais aussi les périodes d'éligibilité courtes ou variables (plus cette dernière est réduite, plus elle oblige à reformuler la demande, donc à engager des démarches) font partie des «barrières administratives » qui, rendant la demande de droit plus complexe, conduisent mécaniquement à une baisse du nombre de demandes formulées.

Aux Etats-Unis, les Etats sont d'ailleurs parfois directement intéressés à une baisse des demandes d'aide par un système de bonus : la baisse des demandes est assimilée à une sortie du dispositif et considérée comme le signe d'un retour à l'emploi. Nombre de travaux anglo-saxons ont mis en évidence un processus de régulation de la demande par les barrières administratives, conduisant qui plus est à une forme d'exclusion administrative [Brodkin, Majmundar, 2010] de certains publics, moins " avantagés administrativement », c'est-à-dire moins en capacité de répondre aux normes institutionnelles.

En France, on assiste à un mouvement contradictoire. A rebours des programmes de simplification administrative annoncés, et sur certains plans mis en place ${ }^{2}$, depuis plus de 20 ans, on constate un accroissement de la complexité des procédures dû une augmentation de la conditionnalité des droits sociaux. II est en effet nécessaire d'apporter de plus en plus de preuves, non seulement matérielles (justificatifs et autres) mais aussi comportementales (prouver sa motivation, sa bonne volonté à s'en sortir) de son éligibilité. Les politiques d'activation, dont le RSA représente le fer de lance, entraînent une multiplication des épreuves de vérification pour les demandeurs mais aussi pour les services aboutissant à ce qu'Elisa Chelles nomme une « bureaucratisation de l'éligibilité " ${ }^{3}$.

Le développement polycentrique (ou multi-acteurs) des politiques qui accroît l'émiettement administratif, a pour conséquence de rendre toujours plus compliqué l'accès aux droits pour les publics et leur application par les services prestataires. Cette organisation générale des politiques conduit nécessairement à ce que leurs «ouputs » concrets et individualisés - en l'occurrence les prestations sociales - soient adossés à de multiples références législatives et réglementaires. Une telle profusion rend plus incertains l'interprétation des règles et le sens du droit dans le traitement des demandes. Cette insécurité juridique pose le problème de la justiciabilité des droits sociaux [Roman, 2007].

Comment les personnes peuvent-elles accéder à leurs droits à différentes prestations quand cette complexité produit toutes les conditions pour qu'elles ne les connaissent pas ou bien abandonnent des démarches devenues des casse-têtes et des parcours du combattant? La question n'est plus seulement celle du non-recours par manque d'information des destinataires, mais aussi celle du nonrecours par non proposition, notamment lorsque les agents des services prestataires ne sont plus en mesure de mettre en œuvre les droits.

Proposer des droits suppose de les connaître. La remarque pourrait paraître triviale s'agissant de professionnels censés gérer l'accès aux droits. Pourtant, la méconnaissance ou la connaissance partielle des droits les concerne aussi. La multiplicité des dispositifs et leur dispersion institutionnelle, les périodes de montée en charge de nouveaux droits ou réglementations, les modifications apportées aux dispositifs, aussi bien que les effets des réorganisations territoriales, ou le fait que certaines institutions communiquent peu sur leurs aides - qu'on pense par exemple au Tarif Première Nécessité ou aux procédures de reconnaissance pour maladie professionnelle - placent les agents de l'accès aux droits dans une position délicate.

\footnotetext{
${ }^{2}$ Notons néanmoins que le plus souvent, les solutions de la simplification administrative paraissent surtout pensées d'un point de vue technologique (télétraitement, télé-déclaration, traitement à distance, etc.), c'est-à-dire là où elles permettent de réaliser des économies de gestion.

${ }^{3}$ "Conditionner une aide sociale génère de véritables coûts bureaucratiques, tant sur le plan des frais de fonctionnement que des dépenses de personnel. Dans une politique sociale conditionnelle, le pauvre pour être secouru, doit apporter la preuve non seulement de son indigence mais également de sa bonne volonté à s'en sortir. La puissance publique doit, pour ce faire, mettre en place une logistique particulière: il s'agit de vérifier la présence d'une motivation. Tâche délicate qui pousse l'action sociale à se re-bureaucratiser. Formulaires, justificatifs, attestations recoupements, informatisation : autant de procédés qui redonnent un large pouvoir aux bureaux. "[Chelles, 2014, 130].
} 
En matière de non-recours par non proposition, la méconnaissance ou la mauvaise connaissance des droits n'explique en effet pas tout. Proposer des droits suppose aussi d'avoir les marges de manœuvre pour le faire ${ }^{4}$. Or les institutions placent souvent les agents prestataires dans une injonction contradictoire, consistant à préconiser un examen global du dossier lors des rencontres avec les ouvrants-droit potentiels, tout en soumettant leur travail à des normes ou des indicateurs de qualité supposant de traiter rapidement les demandes, à leur institution exclusivement [Dubois, 2012]. Le constat de cette contradiction est régulièrement dressé par les travailleurs sociaux : faisant état de leurs difficultés pour calculer les droits et obtenir des interlocuteurs dans les services concernés, ils admettent parfois constituer des dossiers sans avoir l'assurance qu'ils seront finalement éligibles. Acteurs principaux et souvent passages obligés de l'accès aux droits, ils sont malgré eux parfois partie prenante de la production du non-recours.

Au final, si l'empilement des dispositifs et la diversité des portes d'entrée, et à chaque demande la multitude de conditions, obligations et devoirs à respecter, conduit à mettre en cause directement la procédure du ciblage, le problème se situe fondamentalement en amont, dans l'environnement institutionnel et réglementaire des dispositifs proposés. On voit bien dans les études sur le nonrecours combien celui-ci est lié, outre le foisonnement de réglementations difficiles à simplifier et articuler, aux fonctionnements tantôt en silos, tantôt en partenariat, qui complexifient l'accès à des droits souvent connexes. Dans l'ensemble, ce ne sont pas seulement les critères ou les modalités techniques du ciblage qui sont problématiques. En revanche, les systèmes d'informations notamment sur les ressources des ménages - compliquent l'ouverture et la liquidation des droits, ce qui occasionne bien souvent du non-recours ou des ruptures dans les parcours des bénéficiaires.

Tout cela représente des coûts de production très élevés pour les organismes prestataires qu'il serait nécessaire de mesurer de façon systématique dans le cadre d'une politique de lutte contre le nonrecours ${ }^{5}$. Selon nous, les coûts de production découlent moins de l'extension des conditionnalités dont il faudrait encore prouver si et comment elles sont appliquées - que de leur architecture technique. La sélectivité dans l'accès aux ressources répond en premier lieu à l'enjeu du calcul de la " pente incitative ", c'est-à-dire du seuil à partir duquel on estime que les publics visés auront intérêt à entrer dans le dispositif, tout en maîtrisant en contrepoids les dépenses occasionnées par la prestation. Si l'aspect budgétaire entre en ligne de compte du coté des potentielles dépenses imputées à la distribution des prestations, en revanche, les coûts induits par la complexité des dispositifs ne sont pas ou peu évalués. Or cette complexité, qui place les ouvrants-droit potentiels, comme les professionnels, dans une position d'incertitude, a en effet un coût: selon la Branche famille, sur quatre dossiers de RSA reçu, en moyenne un seul est éligible ; les trois quart des dossiers sont donc constitués à perte et représentent une "perte en ligne », depuis l'ouvrant-droit potentiel en passant par l'agent instructeur et les agents des CAF.

Aussi, peut-on s'interroger sur la faisabilité de la solution avancée, visant à mettre en place un formulaire unique de demande pour les minima sociaux, surtout après avoir substitué au système déclaratif avec vérification a posteriori, une vérification a priori. En particulier, ce mode de traitement ne donne aucune prévisibilité de leurs droits aux minima sociaux aux personnes en situation de précarité, inquiètes de voir leurs ressources varier en fonction de changements survenus dans leur situation. C'est pourquoi, parmi les recommandations proposées dans le cadre de la préparation du Plan pluriannuel de lutte contre la pauvreté et l'exclusion sociale, le groupe de travail "Accès aux droits et aux biens essentiels, minima sociaux » avait proposé une annualisation des droits au RSA et aux prestations connexes. On ne retrouve pas de trace de cette proposition dans la liste des recommandations de la mission de l'Inspection générale des affaires sociale (IGAS) chargée de l'évaluation du Plan [Chérèque, Vanackere, 2014].

\section{Démontrer l'efficacité économique des dépenses sociales}

\footnotetext{
${ }^{4}$ Nous laissons ici de coté l'incidence des représentations personnelles et/ou le poids de la culture professionnelle dans les pratiques des agents.

${ }^{5}$ Pour le RSA (socle et activité), les indus représentent 803 millions d'euros en 2012 sur 10,5 milliards de versement total. A-t-on estimé le temps de travail nécessaire à leur traitement, comme à celui du non-recours évalué à 5,3 milliards ?
} 
Les acteurs centraux des politiques sociales ont conscience que mettre fin au ciblage est illusoire. Aucun d'entre eux n'est dans une attente millénariste de la croissance. Tous constatent que celle-ci, au mieux stabilisée aux alentours de $1 \%$ à $1.5 \%$ l'an, ne peut pas constituer le présupposé d'un retour à l'universalité, ni d'ailleurs d'aucun scénario d'avenir. En effet, comment revenir à l'universalité des droits alors que la croissance qui a permis l'accès automatique aux prestations sociales de santé, de chômage et de retraite, n'est plus là depuis longtemps et n'est pas prête de ressurgir ? Ou comment mettre fin aux conditions, obligations et devoirs dans l'accès aux droits, vu le chômage de masse de longue durée et l'emprise des politiques d'exonération fiscale et de baisse du coût du travail censées relancer la croissance ? Dans ce contexte, tout débat sur davantage d'universalité est aussitôt rattrapé par la représentation de la dépense sociale comme seul coût [Djouldem, Saillard, Warin, 2013].

Cette représentation comptable de la dépense publique est un autre enjeu de fond pour la lutte contre le non-recours, et principal pour sa propre suite. Alors que la réduction des déficits publics, notamment par la maîtrise des dépenses sociales, est un impératif que le gouvernement doit respecter pour tenir ses engagements européens, pourquoi donc réduire le non-recours ? D'un point de vue strictement comptable, la lutte contre le non-recours peut apparaître ni plus ni moins comme une «ineptie politique». Elle implique des dépenses supplémentaires et de renoncer aux économies que le phénomène engendre. Elle conduit à se priver d'une aubaine financière de plusieurs milliards d'euros [Odenore, 2013]. Les non dépenses liées au non-recours sont autant de dépenses en moins qui procurent une variable d'ajustement bien utile en période d'austérité budgétaire.

Si l'on revient un instant au Plan pluriannuel, nous pouvons remarquer que le principe de " juste droit » qu'il promeut ne peut se satisfaire de cette approche comptable de la dépense publique. Selon ce principe, chaque citoyen doit avoir accès pleinement et sans retard à l'intégralité des droits auxquels il est éligible. La lutte contre le non-recours est ainsi érigée comme l'un des deux piliers d'une politique générale de l'accès aux droits sociaux ; l'autre pilier étant la lutte contre la fraude aux prestations sociales. Agir pour l'accès aux droits, c'est à la fois éviter des dépenses injustifiées liées à la fraude, mais c'est également agir contre les non-dépenses injustes induites par le non-recours qui pénalisent des populations. C'est ce que le vice-président du Conseil d'État indiquait en affirmant que l'action pour l'accès aux droits est, avec la lutte contre la fraude, l'une des composantes de l'intérêt général [Sauvé, 2011].

Le point de vue comptable s'adosse à une représentation de la dépense sociale comme seul coût. II s'accommode d'un programme de rigueur et d'austérité qui affirme que toute nouvelle augmentation de dépenses doit être couplée et subordonnée à une nouvelle augmentation de recettes, et privilégie des mesures successives d'exonération et de réduction des prélèvements au nom de la compétitivité des entreprises. Mais il a aussi pour conséquence d'occulter deux questions majeures : celle des coûts sociaux, directs et indirects, induits par le non-recours, et celle du possible manque à gagner induit par des dépenses publiques qui ne se font pas. Dit autrement, la lutte contre le non-recours a aussi pour enjeu principal de produire les politiques sociales à partir une vision positive de la dépense publique.

Dans ce sens, plusieurs faits peuvent appeler à se départir du seul point de vue comptable. Les prélèvements obligatoires (impôts et cotisations sociales) ne sont pas que des mécanismes d'extraction de ressources. Les recettes fiscales et sociales servent à financer des services publics et le fonctionnement quotidien du système de prestations et d'aides sociales. En période de crise, les prestations et les aides sociales permettent de compenser les pertes de revenus et de soutenir la demande. Elles contribuent à la création d'emplois dans le secteur de l'économie sociale et solidaire. Elles génèrent des dépenses privées (de salaires et de consommations), lesquelles produisent en retour de nouvelles recettes par le biais de la cotisation et de la fiscalité... dont s'alimentent les budgets de la protection sociale : un cercle vertueux fonctionnant à l'opposé de celui que le Fonds monétaire international (FMI) décrit désormais comme la conséquence des politiques d'austérité qu'il avait si ardemment défendu [Berg, Ostry, Tsangarides, 2014]. A l'inverse, les coupes dans les budgets sociaux anémient les dépenses privées et pèsent ainsi sur l'activité et la croissance. L'impact récessif des politiques de resserrement budgétaires est reconnu. En Grande-Bretagne, le programme d'assainissement des finances publiques du gouvernement Cameron visant à ramener le déficit public de 10,4\% du PIB en 2010 à 1,5\% en 2016 a freiné l'activité, avec une baisse du PIB en 2011 évaluée à moins 0,7 . En France, les politiques que l'on peut qualifier d'austérité (même si la maîtrise des dépenses porte non pas sur leur baisse globale mais sur un ralentissement inégalé de leur progression) ont coûté $0,8 \%$ du PIB en 2010 et 2,2\% en 2013 [Heyer et al., 2013]. Au total, la 
dépense sociale, lorsqu'on l'intègre dans l'ensemble du circuit, remplit une fonction économique plus vertueuse que les épargnes des ménages qui soutiennent des comportements rentiers ou alimentent des spéculations financières.

En réhabilitant les fonctions positives de la dépense sociale, la contradiction entre la progression vers l'égalité de l'accès aux droits et le respect des contraintes économiques peut s'effacer en partie. Le non-recours n'apparaît plus alors comme une opportunité d'économie budgétaire, d'autant plus efficace qu'elle est peu visible. II est plutôt la marque d'un échec des politiques sociales et l'indice d'une destruction massive de richesses. La lutte contre le non-recours peut prétendre à une efficacité économique. Son défi n'est donc pas que social, il est aussi économique.

Ce double défi, par conséquent, attend les acteurs des politiques sociales, qui sont redevables de l'efficacité des dépenses publiques. Mais c'est aussi celui de la recherche sur le non-recours, dont la mission de l'IGAS chargée de l'évaluation du Plan pluriannuel préconise le développement dans plusieurs de ses recommandations. Après avoir mesuré et caractérisé le phénomène, produit des typologies explicatives, le principal enjeu en termes de production de connaissances est de développer maintenant une approche économique du non-recours. De nouvelles recherches pourraient se fixer plusieurs orientations : mesurer précisément les non-dépenses et connaître leurs usages ultérieurs ; identifier et mesurer en termes monétaires ou pas la diversité des coûts sociaux directs et indirects pour les non requérants comme pour les services prestataires ; estimer les effets multiplicateurs ${ }^{6}$ perdus pour la collectivité du fait du non-recours et en même temps l'efficience allocative ${ }^{7}$ des dépenses publiques ; modéliser les changements de comportements des bénéficiaires survenant après une modification d'une ou de plusieurs conditions ; ... Une telle recherche, démonstrative et pédagogique, pourrait en même temps être fort utile à la formation de bon nombre de cadres qui dans les administrations, les organismes sociaux et les collectivités publiques ont ou auront à mettre en œuvre et à évaluer les résultats des plans d'actions de la lutte contre le nonrecours. Bien menée, cette recherche serait aussi au service de la correction nécessaire des situations de sous-productivité ou de dépenses inefficaces [Fragonard, 2012], que la lutte contre le non-recours ne doit en rien évacuer.

\section{Conclusion}

Les enjeux de la lutte contre le non-recours pour la production des politiques sociales présentés ici sont évidemment à discuter. La légitimité des motifs des politiques, la sécurisation des droits, la conception de la dépense sociale sont des questions difficiles à formuler. Et ce ne sont pas les seules: on a pu récemment indiquer comment au local l'action sur le non-recours pouvait se confronter à certaines représentations sociales de l'assistance [Warin, 2014]. Le point de vue exprimé a donc pour vocation à être remanié et prolongé.

Pour autant, en cherchant à débattre des enjeux de la lutte contre le non-recours pour la production des politiques publiques, nous affirmons l'idée que la lutte contre le non-recours existera essentiellement au travers des changements qu'elle pourra produire dans la manière de construire et de réaliser les politiques sociales. Son existence est liée à sa portée réformatrice. Elle ne peut avoir d'autre perspective selon nous, à moins de disparaître, comme d'autres projets politiques, dans une seule gymnastique verbale. Ce que les politologues anglo-saxons appellent "isomorphic mimicry " pour signifier des politiques ou des plans d'actions qui donnent l'air de faire des réformes sans réellement les produire. Espérons qu'il en soit autrement avec la lutte contre le non-recours.

\section{Bibliographie}

Berg J., Ostry D., Tsangarides C.-G. (2014), "Redistribution, inequality and growth", IMF Staff Discussion Note 14/02. (http://www.imf.org/external/pubs/ft/sdn/2014/sdn1402.pdf)

\footnotetext{
${ }^{6}$ Quand les dépenses publiques produisent des dépenses privées qui abondent en retour les recettes publiques.

L'efficience allocative se réfère à la relation entre les ressources investies dans une politique publique et les effets obtenus. II décrit ainsi le rapport entre les coûts et les bénéfices, également financiers, d'une politique.
} 
Brodkin E.-Z., Majmundar M. (2010), "Administrative Exclusion: Organizations and the Hidden Costs of Welfare Claiming" , Journal of Public Administration Research and Theory (20): 827-848 http://jpart.oxfordjournals.org/content/20/4/827.full.pdf+html

Chelles E (2012), Gouverner les pauvres. Politiques sociales et administration du mérite, Presses universitaires de Rennes.

Chérèque F., Vanackere S. (2014), Evaluation de la $1^{\text {ère }}$ année de mise en œuvre du plan pluriannuel contre la pauvreté et pour l'inclusion sociale, Paris, IGAS.

(http://www.igas.gouv.fr/spip.php?article353)

Conning J., Kevane M. (2002), "Community based targeting mechanisms for social safety nets: a critical review", World Development, 30(3): 375-394.

Djouldem M., Saillard Y., Warin P., (2013), "Lutter contre le non-recours: un révélateur du renouvellement des politiques publiques, in Odenore, L'envers de la fraude sociale. Le scandale du non-recours aux droits et services, Paris, La Découverte : 185-201.

Domingo P. (2012), « Les non-recourants au RSA », L'e-ssentiel (124).

(http://www.caf.fr/sites/default/files/cnaf/Documents/Dser/essentiel/124 essentiel - nonrecours rsa.pdf)

Dubois V. (2010), "Politiques au guichet, politique du guichet », in Borraz O., Guiraudon V. (dir.), Politiques publiques - 2, Changer la société, Paris, Presses de Sciences-Po : 265-286.

Fragonard B. (2012), Vive la protection sociale !, Paris, Odile Jacob.

Gwatkin D. (2000), The Current state of knowledge about targeting the health programs to reach the poor, Washington DC, World Bank.

Heyer E., Cochard M., Ducoudré B., Péléraux H. (2014), "France: moins d'austérité, plus de croissance », Revue de l'OFCE. (http://www.ofce.sciences-po.fr/pdf/revue/3-130.pdf)

Milet H., Warin P. (2014), "La prise en compote es publics dans l'évaluation des politiques publiques », Revue française d'administration publique (148) : 991-1002.

ODENORE, (2013), L'envers de la fraude sociale. Le scandale du non-recours aux droits sociaux, Paris, La Découverte.

Roman D. (2007), "Les droits sociaux : des droits à part entière ? Eléments pour une réflexion sur la nature et la justiciabilité des droits sociaux ", in Du Cheyron P., Gelot D., Droit et pauvreté. Contributions issues du séminaire ONPES-DREES-MIRe 2007, Paris : 39-58.

(http://www.onpes.gouv.fr/lMG/pdf/livre entier web.pdf)

Sauvé J.-M. (2011), "Ouverture des entretiens du Conseil d'Etat: Fraudes et protection sociale », Droit social (5) : 481-485.

Sen A. (1995), "The political economy of targeting", in Van de walle D, Nead K (eds.), Public spending and the poor: theory and evidence, Baltimore, The World Bank by the Johns Hopkins University Press: 11-24.

Serverin E. (2012), "Causes et effets du non-recours au RSA-activité », Revue de Droit Sanitaire et Social (4) : 637-645.

Warin P. (2014) «L'action sur le non-recorrs devant des résistances du travail social », Revue Française des Affaires Sociales (1-2) : 64-77. 